\title{
Influence of initial water content of aggregates on the compressive strength and the shrinkage of High Performance Concretes
}

\author{
Abdelkadir MAKANI ${ }^{1}$ and Thierry VIDAL $^{2}$ \\ ${ }^{1}$ Université TAHRI Mohamed Béchar, Département de Génie Civil, LFGM (Laboratoire de Fiabilité du Génie Mécanique), \\ BP 417 Béchar (08000), Algérie \\ ${ }^{2}$ Université de Toulouse, UPS, INSA, LMDC (Laboratoire Matériaux et Durabilité des Constructions), 135, Avenue de \\ Rangueil, F-31 077 TOULOUSE Cedex 4, France
}

\begin{abstract}
The time-dependent deformations of concretes, particularly in the case of high performance concrete (HPC) must be taken into account precisely when measurement of civil engineering works. theses deformations is essential for predicting the deflections, the stress distribution in statically indeterminate structures and the loss of pre-stressing force in elements of pre-stressed concrete. However, the experience shows a certain dispersion of values of instantaneous and delayed deformations measured and some significant differences with the values calculated using the building codes. The objective of the present paper focuses on the parameter which is not taken into account in building codes, which could causes the inaccuracies of their predictions: the initial water content of aggregates. The experimental program includes a comparative study of the mechanical behaviours (instantaneous deformations and shrinkage) of HPC with the same basic formulation (water cement ratio, volume of paste), differing principally in the initial water content of aggregates. The experimental results show that HPC made with wet aggregates has a higher resistance than others (dry aggregates). The pre-saturated aggregates would be a water reservoir in which the dough could slow the self-drying due to hydration in the capillary pores at the level of the Interfacial Transition Zone (ITZ). This would increase the amount of hydrates and improve the mechanical behaviour of this interface through better adhesion and less porosity. If the evolution of shrinkage of HPC with wet or dry aggregates can be distinct during the first months, the effect of this formulation parameter is not clear since it differs according to the type of aggregate. Moreover, in the long term, convergent shrinkage shows that this influence becomes insignificant. The comparisons with estimations of Eurocode 2 model were also performed and showed significant differences with the experimental values.
\end{abstract}

\section{Introduction}

Les granulats est un des paramètres influant du comportement macroscopique du béton [1-3], plus particulièrement le comportement mécanique instantané et différé à long terme [4]. Cependant, leur influence est mal prise en compte dans les divers règlements en vigueur et pourrait être à l'origine des imprécisions de leurs prédictions. Ceci peut également s'expliquer par la difficulté à estimer et découpler l'impact des nombreuses caractéristiques des granulats sur les propriétés physiques et mécaniques des bétons : nature minéralogique, géométrie/forme, porosité, absorption capillaire, performances mécaniques [5].

L'étude présentée dans cet article a pour objectif d'apporter des informations relatives à l'influence de la teneur en eau initiale des granulats sur les déformations instantanées et différées sans charge des bétons.
Le programme expérimental a été mené sur des Bétons à Hautes Performances (BHP) pour lesquels la connaissance précise du comportement mécanique est primordiale en vue d'un dimensionnement correct des ouvrages. Deux types de granulats (siliceux et calcaire) ont été utilisés dans la production de ces bétons de formulations semblables afin d'étudier l'effet de la teneur en eau initiale des granulats sur la résistance en compression et le retrait. 


\section{Matériaux et méthodes expérimentales}

\subsection{Caractérisations des matériaux de base et formulations}

Au cours de la caractérisation expérimentale, un sable unique siliceux roulé $(\mathrm{SnR})$, commun à toutes les formulations des BHP étudiés, et deux types de gravillons de nature minéralogique différente ont été testés. Ces granulats seront nommés par la suite selon la terminologie introduite au Tableau 1 qui présente quelques paramètres physiques et mécaniques permettant de caractériser les différents types de granulat.

Tableau 1. Récapitulatif des propriétés physiques et mécaniques des différents granulats utilisés.

\begin{tabular}{|c|c|c|c|}
\hline \multirow{2}{*}{ Forme } & Sable & \multicolumn{2}{c|}{ Gravillons } \\
\hline Nature & \multicolumn{2}{|c|}{ Roulé } & Concassé \\
\hline Classe & $0 / 4$ & $4 / 10$ & $4 / 12,5$ \\
\hline Nomenclature & $\mathrm{SnR}$ & $\mathrm{SiR}$ & $\mathrm{CaC}$ \\
\hline Densité & \multicolumn{2}{|c|}{2,8} & 2,7 \\
\hline Absorption (\%) & 1,7 & 1,2 & 1,3 \\
\hline Porosité totale (\%) & 8,5 & 5,3 & 4,6 \\
\hline Résistance (MPa) & - & - & 224 \\
\hline Module (GPa) & - & - & 80 \\
\hline
\end{tabular}

Les différents BHP étudiés sont constitués d'un des deux gravillons ( $\mathrm{SiR}$ et $\mathrm{CaC}$ ) et d'un même sable de classe 0/4 (SnR) pour l'état sec $(\mathrm{S})$ et humide $(\mathrm{H})$. Le liant est constitué d'un ciment CEM I 52,5R associé à de la fumée de silice $\left(405+45 \mathrm{~kg} / \mathrm{m}^{3}\right)$. Pour assurer une maniabilité suffisante, un superplastifiant est ajouté à l'eau. Le rapport $\mathrm{E} / \mathrm{L}(0,35)$ et de dosage en granulats (gravillon, $1133 \mathrm{~kg} / \mathrm{m}^{3}+$ sable, $944 \mathrm{~kg} / \mathrm{m}^{3}$ ) sont maintenus constants.

\subsection{Méthodes expérimentales}

Les échantillons coulés étaient des cylindres 11,8x23,6 $\mathrm{cm}$. Six étaient destinés aux mesures de résistance mécanique en compression pour chacune des échéances, à savoir 3 et 28 jours et six pour le retrait. Après le coulage, les moules sont recouverts par un film plastique afin d'empêcher un départ de l'eau libre du béton par évaporation. Ils sont ensuite conservés dans une salle à 20 ${ }^{\circ} \mathrm{C} \pm 1{ }^{\circ} \mathrm{C}$ et $95 \pm 5 \%$ d'humidité relative pendant 24 heures. Le démoulage a été effectué à un jour.

La caractérisation mécanique a été effectuée à 3 et 28 jours à l'aide d'une presse de $3000 \mathrm{kN}$ de capacité maximale, asservie en force avec une vitesse de chargement de $0,5 \mathrm{MPa} / \mathrm{s}$. La résistance en compression a été évaluée suivant les recommandations RILEM 1972.
Pour les mesures de déformations différées sans charge, deux modes de conservation ont été étudiés. Trois éprouvettes sont conservées en mode endogène (sans échange hydrique) dès le démoulage et trois autres en mode dessiccation. Les déformations de retrait sont relevées dès le premier jour après coulage à l'aide d'un rétractomètre (Figure 1).

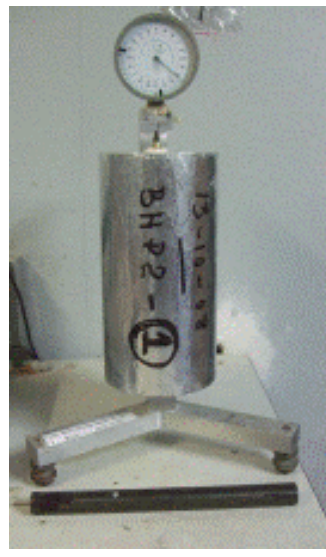

(a)

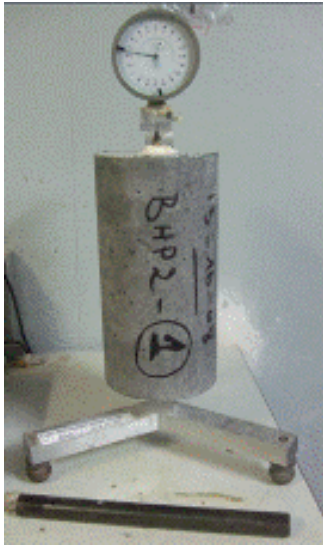

(b)
Fig. 1. Dispositif de mesure des formations de retrait; (a) en mode endogène et (b) en mode dessiccation.

\section{Résultats et discussions}

\subsection{Comportement mécanique instantané}

Les caractéristiques mécaniques instantanées des bétons à 3 jours et à 28 jours sont présentées dans le Tableau 2.

Tableau 2. Récapitulatif des résistances mécaniques en compression.

\begin{tabular}{|c|c|c|c|c|c|}
\hline & Temps (j) & SiRH & SiRS & CaCH & CaCS \\
\hline \multirow{2}{*}{$\begin{array}{c}\text { fcm } \\
(\mathrm{MPa})\end{array}$} & 3 & 54,5 & 54,4 & 66,7 & 56,2 \\
\cline { 2 - 6 } & 28 & 82,2 & 73,5 & 94,7 & 79,8 \\
\hline
\end{tabular}

Les résistances en compression les plus élevées sont mesurées pour le béton avec granulats calcaires. Ce résultat pourrait être corrélé à la plus forte résistance de ces granulats [6]. L'obtention d'une résistance en compression élevée du béton ne semble donc pas donc uniquement assujettie à celle du granulat utilisé. La qualité de l'interface granulat/pâte de ciment pourrait jouer un rôle [7-9]. Ce constat est relativement proche de celui réalisé par de Larrard et Belloc, à savoir que la résistance en compression des bétons est conditionnée principalement par l'interface entre les deux phases pour les classes de résistance faibles à modérées et par la contrainte ultime en compression du granulat dans le cas où elle se rapproche de celle du béton [6] 
La comparaison des résultats des formulations avec granulats préalablement secs ou humides nous permet d'étudier l'influence du paramètre du degré de saturation des granulats sur les performances mécaniques des bétons. Les bétons confectionnés avec des granulats humides ( $\mathrm{SiRH}$ et $\mathrm{CaCH}$ ) présentent une résistance plus élevée qu'avec des granulats secs ( $\mathrm{SiRS}$ et $\mathrm{CaCS}$ ), cet effet étant plus marqué à 28 qu'à 3 jours. Selon Pigeon et al. [10] et Lopez et al. [11], les granulats saturés représenteraient en quelque sorte un réservoir dans lequel la pâte peut puiser pour ralentir l'auto-dessiccation due à l'hydratation dans les pores capillaires situés au niveau de l'interface. En outre, l'utilisation d'un rapport $\mathrm{E} / \mathrm{L}$ réduit de nos formulations dans le but d'atteindre des hautes performances mécaniques pourrait entraîner un déficit d'eau nécessaire à l'hydratation localement et notamment au niveau de la zone de transition (ITZ). Dans le cas des granulats humides, l'eau relâchée permettrait d'augmenter la quantité d'hydrates et d'améliorer la résistance de cette interface grâce à une meilleure adhérence. Cette observation conforte la potentielle influence de la qualité de l'ITZ sur la résistance en compression des bétons. Conformément aux cinétiques d'hydratation, cet effet serait donc plus effectif à 28 qu'à 3 jours.

\subsection{Comportement différé sans charge en mode endogène}

La Figure 2 présente les évolutions des valeurs moyennes des déformations longitudinales de retrait endogène des bétons à base des granulats siliceux et calcaires humides et secs ainsi que les prédictions de l'Eurocode 2 [12].

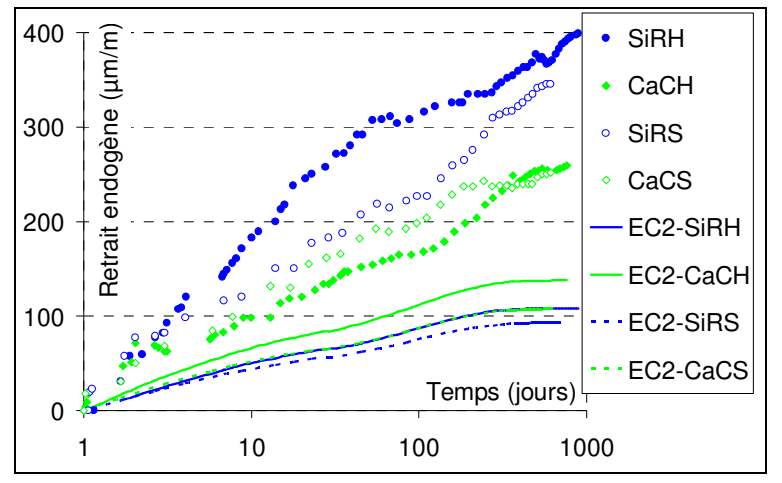

Fig. 2. Effet de la teneur en eau des granulats sur les retraits endogènes des bétons à base des granulats siliceux et calcaires.

On constate des comportements assez différents suivant l'état hydrique initial des granulats. Cependant, l'effet de la teneur en eau n'est pas le même suivant la nature du granulat. Le BHP avec granulat siliceux humide développe le retrait endogène le plus intense au jeune âge comparativement à celui avec granulats secs. Mais l'ordre est inversé pour les BHP avec granulats calcaires. Néanmoins, ces évolutions de déformations assez dispersées durant les premiers mois tendent à se rapprocher aussi bien en termes de cinétiques que d'amplitudes au bout d'un an pour chaque nature minéralogique de granulat.

Il est généralement admis que les granulats, de par leur porosité ouverte, peuvent être considérés comme des réservoirs d'eau pouvant capter ou relâcher de l'eau dans la pâte. Ainsi, les granulats secs absorbent de l'eau pendant le gâchage et une quantité moindre d'eau reste disponible pour l'hydratation à court terme. Ceci implique une structure plus dense de la pâte de ciment contenant des pores plus fins (à un degré d'hydratation donné). Les forces capillaires sont donc plus intenses, ce qui se traduit par un retrait d'auto-dessiccation plus élevé. Une autre explication avancée par certains chercheurs [10-11] et [13] serait que les granulats humides emmagasinent de l'eau et la fournissent plus tard au ciment quand l'eau de gâchage n'est plus disponible pour l'hydratation. Cette eau sera alors utilisée par le ciment pour poursuivre son hydratation. Les deux mécanismes se produisent probablement à des moments différents. Ces transferts d'eau sont probablement complexes et variables car fonctions de plusieurs paramètres interdépendants comme la capacité d'absorption des granulats, la porosité ouverte, la qualité de l'ITZ certainement variable selon la nature minéralogique du granulat (épitaxie pour le granulat calcaire). Ils se produisent au jeune âge principalement durant la phase d'hydratation et des réactions pouzzolaniques de la fumée de silice, lorsque la quantité d'eau non liée est encore importante. Cela engendrerait des différences d'évolutions de retrait endogène. Mais ces phénomènes ne semblent finalement modifier que les cinétiques et non le potentiel de déformation de retrait endogène à plus long terme.

La Figure 2 met en évidence des sous-estimations importantes de l'Eurocode 2. Bien que ce modèle prévoie des écarts dus aux variations des résistances en compression, les différences entre valeurs expérimentales sont plus fortement marquées. On peut en déduire que d'autres paramètres non pris en compte interviennent. Étant donné que les paramètres intrinsèques aux formulations ont été fixés, l'influence du paramètre granulat semble avérée.

\subsection{Comportement différé sans charge en mode dessiccation}

La Figure 3 présente les évolutions des valeurs moyennes des déformations longitudinales de retrait total des bétons à base de granulats siliceux et calcaires, humides et secs ainsi que les prédictions de l'Eurocode 2[12].

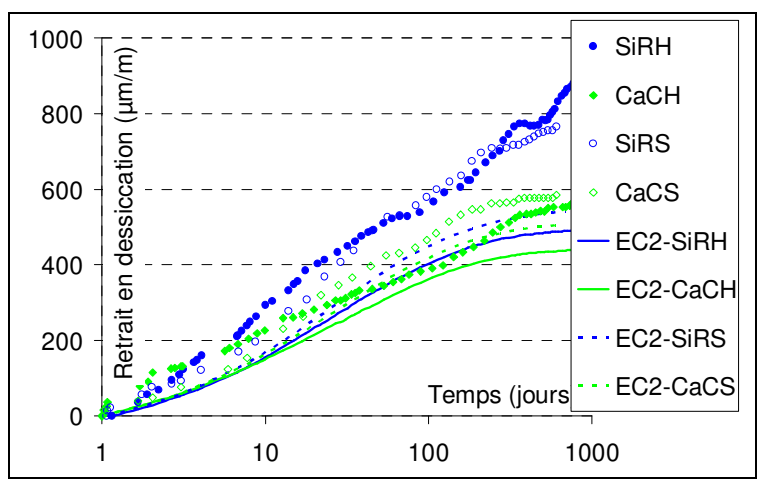

Fig. 3. Effet de la teneur en eau des granulats sur les retraits totaux des bétons à base des granulats siliceux et calcaires. 
D'après la Figure 3, les différences entre les évolutions de retrait total sont plus faibles que celles détectées à court terme en mode endogène. A long terme, elles deviennent négligeables quelque soit la nature minéralogique du granulat.

Le fait que les granulats humides fournissent de l'eau à un moment donné au mélange pourrait expliquer les légères variations de comportement durant la phase d'hydratation et des réactions pouzzolaniques. Mais à long terme et à l'échelle de l'éprouvette, cette influence est peu perceptible.

Enfin, l'Eurocode 2 sous-estime le retrait total pour toutes les configurations (Figure 3) et démontre également des imprécisions en n'intégrant que les paramètres de résistance en compression.

\section{Conclusions}

Cette étude présente le comportement mécanique instantané et différé sans charge des BHP ne se différenciant que par la nature minéralogique et la teneur en eau initiale des granulats. Malgré que les différents bétons aient même formulation de base, les résultats montrent parfois d'importantes différences.

La résistance en compression semble conditionnée par la qualité de l'interface pâte-granulat (ITZ), avec notamment les valeurs plus fortes observées pour le BHP avec granulats calcaires probablement grâce au phénomène d'épitaxie.

Les BHP confectionnés avec des granulats humides présentent une résistance plus élevée que ceux avec des granulats secs. Les granulats saturés représenteraient en quelque sorte un réservoir d'eau dans lequel la pâte pourrait puiser pour ralentir l'auto-dessiccation due à l'hydratation dans les pores capillaires situés au niveau de l'ITZ. Cela permettrait d'augmenter la quantité d'hydrates et d'améliorer la résistance de cette interface grâce à une meilleure adhérence et une moindre porosité.

La comparaison entre les retraits des bétons à base de granulats humides et secs montre que les évolutions de déformations, qui sont assez dispersées durant les premiers mois, tendent à se rapprocher, au bout d'un an, aussi bien en termes de cinétique que d'amplitude pour chaque nature minéralogique de granulat. Le fait que les granulats humides fournissent de l'eau, à un moment donné, au mélange pourrait expliquer les légères variations de comportement durant la phase d'hydratation, y compris les réactions pouzzolaniques. Mais à long terme et à l'échelle de l'éprouvette, cette influence paraît peu perceptible.

De manière générale, les prédictions du modèle Eurocode 2 sous-estiment les déformations de retrait et ne permettent pas de retrouver les écarts entre les amplitudes des différents BHP. Cette étude démontre ainsi les limites de ce modèle dans l'estimation du comportement mécanique à long terme de ces matériaux.

\section{References}

1. A. M. Neville, Properties of Concrete. Fourth and Final Edition, John Wiley \& Sons, Inc, New-York, USA (1996)

2. G. Pons et J. M. Torrenti, Retrait et fluage. La durabilité des bétons, Bases scientifiques pour la formulation de bétons durables dans leur environnement. Sous la direction de Jean-Pierre OLLIVIER et Angélique VICHOT, Presses de l'ENPC, Paris, France (2008)

3. A. Makani, T. Vidal, G. Pons et G. Escadeillas, Time-dependent behaviour of high performance concrete: influence of coarse aggregate characteristics, ICEM14 International Conference on Experimental Mechanics, Poitiers, France, 4-9 July (2010)

4. A. Makani, T. Vidal et G. Pons, Influence de la nature minéralogique des granulats sur le comportement mécanique différé des bétons, Colloque International Francophone NoMaD, Toulouse, France, 19-20 Novembre (2012)

5. A. Makani, Influence de la nature minéralogique des granulats sur le comportement mécanique différé des bétons, Thèse de doctorat, INSA, Toulouse, France (2011)

6. F. de Larrard et A. Belloc, The Influence of Aggregate on the Compressive Strength of Normal and High-Strength Concrete, ACI Materials Journal, 94, 5, 417-426 (1997)

7. J. P. Ollivier, J. C. Maso, et B. Bourdette, Interfacial transition zone in concrete, Advanced Cement Based Materials, 2, 1, 30-38 (1995)

8. J. C. Maso, Interfacial transition zone in cement, E\&FN Spon., 108. London, England (1996)

9. I. Gaweska Hager, Comportement à haute température des bétons à haute performanceévolution des principales propriétés mécaniques, Thèse de doctorat, ENPC, Paris, France (2004)

10. M. Pigeon, G. Toma, J. Marchand et B. Bissonnette, Étude expérimentale du retrait endogène restreint au jeune âge, Mater. Struct., 36, 264 ,666-672 (2003)

11. M. Lopez, L. F. Kahn et K. E. Kurtis, Effect of Internally Stored Water on Creep of HighPerformance Concrete, ACI Materials Journal, 105, 3, 265-273 (2008)

12. Eurocode 2, EN 1992-2, Design of concrete structures. Concrete bridges, (2006)

13. N. Belaribi, G. Pons et B. Perrin, Delayed behaviour of concrete: Influence of additions and aggregate characteristics in relation to moisture variations, Cement and Concrete Research, 27,9, 1429-1438 (1997) 